\title{
Simulation of strain fields in GaSb/InAs heteroepitaxial system
}

\author{
S.V. Shutov', Ye.A. Baganov ${ }^{2}$ \\ ${ }^{1}$ V. Lashkaryov Institute of Semiconductor Physics, NAS of Ukraine \\ 41, prospect Nauky, 03028 Kyiv, Ukraine \\ E-mail: shutov_sv@mail.ru \\ Phone/Fax $+3 \overline{8}$ (044) 5255457 \\ ${ }^{2}$ Kherson National Technical University, 24, Berislavskoye shosse, 73008 Kherson, Ukraine \\ E-mail:ewgb@newmail.ru,phone:+380(552) 326922
}

\begin{abstract}
Mechanical strains taking place in $\mathrm{GaSb} / \mathrm{InAs}$ heterosystem in the presence of misfit dislocation network are investigated. Distributions of energy of strains and deformations in the system with misfit dislocation network were found using twodimensional simulation. The radius of the dislocation core, depths of the strain penetration into the substrate and epitaxial layer as well as change of the material bandgaps near the heterointerface were calculated.
\end{abstract}

Keywords: misfit dislocation, strain distribution, GaSb, InAs, heteroepitaxy.

Manuscript received 09.01.06; accepted for publication 29.03.06.

\section{Introduction}

In recent years, properties of broken gap II type heterojunctions based on InAs and GaSb solid solutions are widely used for production of different optoelectronic devices [1]. Also the GaSb/InAs heterojunction is very promising for manufacturing the tandem thermophotovoltaic converters, because there is no need to form a tunnel diode between an upper (based on $\mathrm{GaSb}$ ) and lower (based on InAs) cascades [2].

Misfit of lattice parameters $(\approx 0.7 \%)$ leads to forming the network of misfit dislocations at the heterointerface. The availability of dislocations causes appearing the non-uniform fields of elastic strains, local change of bandgap and appearing the charge regions near a dislocation core [3]. All these defects can significantly decrease a quality of devices, manufactured using these structures. That is why, the aim of this work is calculation of strain fields that appear around dislocations in the GaSb/InAs heterosystem.

\section{Calculation model}

As a base for these calculations, the Van-der-Merwe approach was used [4]. According to this model, it assumed that an equilibrium position for the epitaxial layer atom (or finite element) is the position when an upper atom is located just over the lower one. A dislocation appears when displacement between atoms of the epitaxial layer and substrate reaches a half of the lattice parameter (Fig. 1). The distance between misfit dislocations was taken in accord with [4]

$l_{d}=\frac{a_{s} a_{l}}{a_{l}-a_{s}}$,

where $a_{s}, a_{l}$ are the lattice parameters of substrate and epitaxial layer materials, respectively. The structure with misfit dislocation network is periodic with the period $l_{d}$ and symmetric with respect to $x=0$. That is why, the region of simulation was limited by $x \in\left[0 ; l_{d} / 2\right]$.

Strain and deformation distributions in the considered structure are described by the following set of equations:

$$
\left\{\begin{array}{l}
\nabla^{4} \chi=0 ; \sigma_{x x}=\frac{\partial^{2} \chi}{\partial y^{2}} ; \sigma_{x y}=-\frac{\partial^{2} \chi}{\partial x \partial y} ; \sigma_{y y}=\frac{\partial^{2} \chi}{\partial x^{2}} \\
u_{x x}=\frac{1}{E(y)}\left(\sigma_{x x}-\mu(y) \sigma_{y y}\right) ; u_{y y}=\frac{1}{E(y)}\left(\sigma_{y y}-\mu(y) \sigma_{x x}\right) \\
u_{x y}=\frac{1+\mu(y)}{E(y)} \sigma_{x y}
\end{array}\right.
$$

with the boundary conditions 


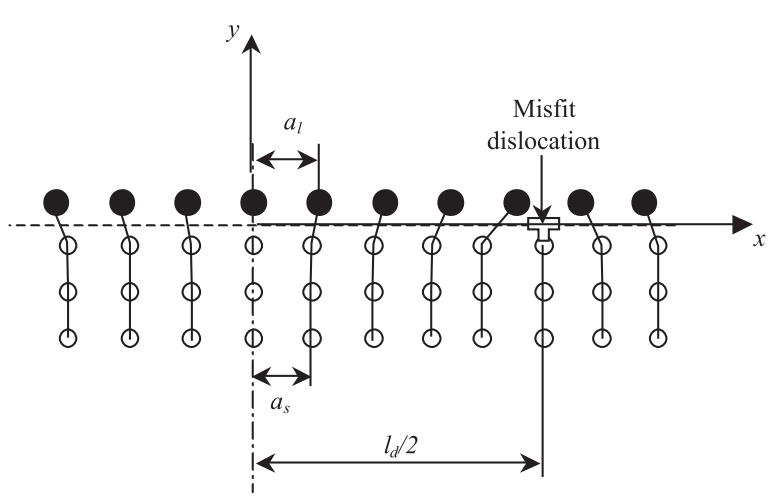

Fig. 1. Model of misfit dislocation formation.

$$
\left\{\begin{array}{l}
\sigma_{y y}(x, h)=0 ; \sigma_{x y}(x, h)=0 ; \sigma_{x y}(x,+0)= \\
=f_{x}(x) ; \sigma_{x y}(x,-0)=-f_{x}(x) ; \\
\sigma_{y y}(x,+0)=f_{y}(x) ; \sigma_{y y}(x,-0)=-f_{y}(x) ; \\
\sigma_{x x}(x,-\infty)=0 ; \sigma_{y y}(x,-\infty)=0 ; \\
\sigma_{x y}(x,-\infty)=0 ; u_{x}(0, y)=0 ; u_{x}\left(l_{d} / 2, y\right)=0 .
\end{array}\right.
$$

Here $\chi$ is the strain function, $\sigma_{i, j}, u_{i, j}$ are the components of tensors for strains and deformations, respectively, $u_{x}$ is the $x$-component of the displacement vector, $h$ is the thickness of the epitaxial layer, $f_{x}(x)$, $f_{y}(x)$ are the $x$ - and $y$-components of the specific force of atom interaction at the heterointerface. $E(y)$ and $\mu(y)$ are Young's module and Poisson's ratio that are defined by the following expressions:

$$
\begin{aligned}
& E(y)= \begin{cases}E_{\mathrm{GaSb}}, & y \geq 0 \\
E_{\mathrm{InAs}}, & y<0\end{cases} \\
& \mu(y)= \begin{cases}\mu_{\mathrm{GaSb}}, & y \geq 0 \\
\mu_{\mathrm{InAs}}, & y<0\end{cases}
\end{aligned}
$$

where $E_{\mathrm{GaSb}}, E_{\mathrm{InAs}}, \mu_{\mathrm{GaSb}}, \mu_{\mathrm{GaSb}}$ are Young's modules and Poisson's ratios of GaSb and InAs, respectively, which have been calculated from the elastic constants [5] (for GaSb: $\quad C_{11}=88.49 \mathrm{GPa}, \quad C_{12}=40.37 \mathrm{GPa}, C_{44}=$ $43.25 \mathrm{GPa}$; for InAs: $C_{11}=83.29 \mathrm{GPa}, C_{12}=45.26 \mathrm{GPa}$, $C_{44}=39.59 \mathrm{GPa}$ ). The latter two equations of (2) describe the system symmetry. To realize the simulations of the deformation distribution described by the equations (1) (4), the method of finite elements [6] was used. But using the forces of atom interaction $f_{x}(x)$ and $f_{y}(x)$ in this simulation in accord with any adequate atom interaction model (for instance, the Peierls-Nabarro model [7]) led to a nonlinear or, depending on the accepted interaction model, transcendent equation set resulting in significant complication of the calculations. To avoid a necessity of consideration of interaction at the heterointerface, an intermediate layer with the thickness in a finite element was introduced between the substrate and epitaxial layer. Physical parameters of the intermediate layer were taken as averages of relative parameters of the epitaxial layer and substrate. Initial non-equilibrium state has been chosen as follows. All deformations are localized in the intermediate layer. The substrate and epitaxial layer are unstrained, and the intermediate layer is deformed in such a way to fit one its side, which is in contact with the epitaxial layer, to the epitaxial layer lattice and the other side to the substrate lattice simultaneously (see Fig. 2). Dotted lines and arrows show positions of intermediate layer atoms in the unstrained state and their displacement directions.

The initial displacement of nodal points of the intermediate layer elements with respect to their equilibrium states was defined as follows:

$u_{x 0 i}=\Delta_{x} \frac{a_{l}-a_{s}}{a_{l}+a_{s}}\left(i_{\max }-i\right)$

for the interface of the intermediate layer - epitaxial one and

$u_{x 0 i}=-\Delta_{x} \frac{a_{l}-a_{s}}{a_{l}+a_{s}}\left(i_{\max }-i\right)$

for that of intermediate layer - substrate, where $\Delta_{x}$ is the dimension of the finite element of the intermediate layer along the $O x$-direction, $i$ is the number of finite elements, $i_{\max }$ is the quantity of finite elements along the $O x$-direction.

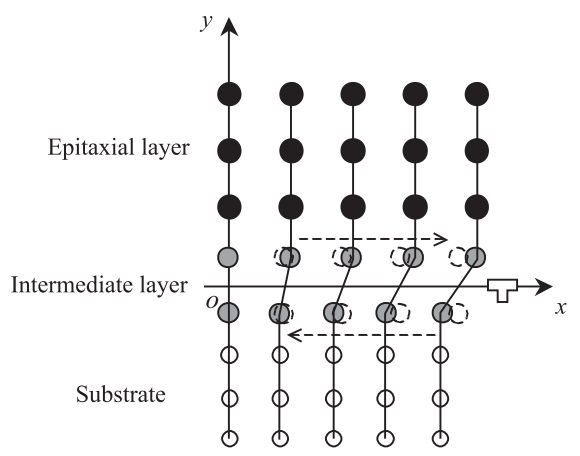

Fig. 2. Initial state of the structure for simulation with the method of finite elements.

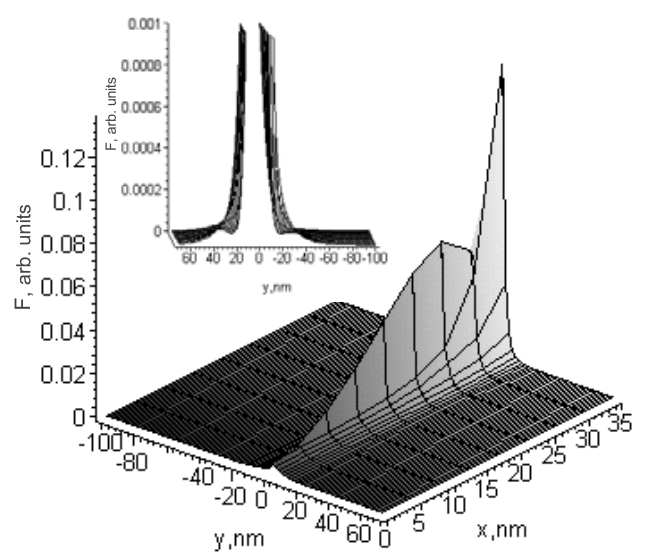

Fig. 3. Distribution of strain energy $F(x, y)$ in $\mathrm{GaSb} / \mathrm{InAs}$ heterostructure with misfit dislocation network. Insertion contains the distribution of strain low energies. 


\section{Results and discussion}

As a result of the calculations, the dependence of the strain energy distribution $F(x, y)$ was obtained. Distribution of the strain energy for the $70 \mathrm{~nm}$ thick epitaxial layer of is shown in Fig. 3. Insertion in Fig. 3 represents a distribution of low strain energies. Distributions of the components of the strain tensor $\sigma_{x x}(x, y), \sigma_{y y}(x, y), \sigma_{x y}(x, y)$ are shown in Fig. $4 \mathrm{a}, \mathrm{b}, \mathrm{c}$, respectively.
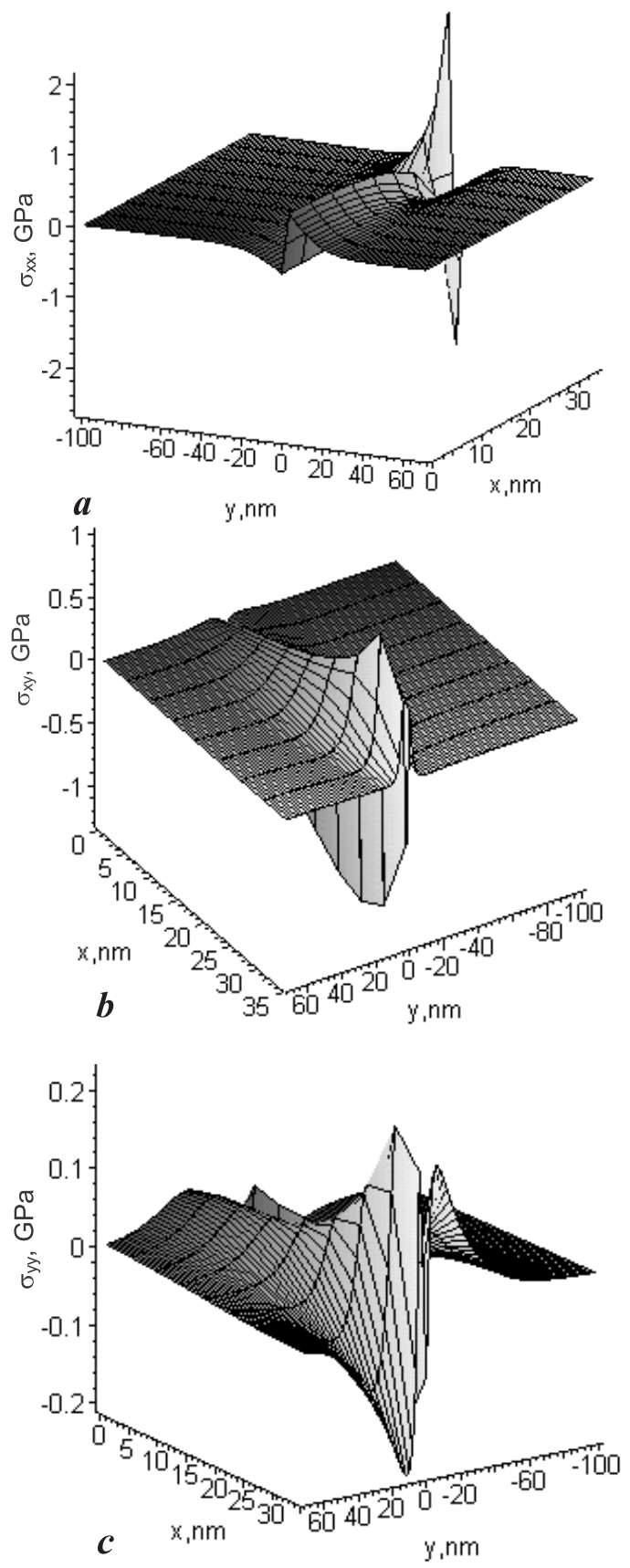

Fig. 4. Distribution of strains in the $\mathrm{GaSb} / \mathrm{InAs}$ heterostructure with the misfit dislocation network. Components of the strain tensor: $\sigma_{x x}(x, y)(\mathrm{a}), \sigma_{x y}(x, y)(\mathrm{b}), \sigma_{y y}(x, y)(\mathrm{c})$.
Based on the simulation results, it can be found that strains are localized near the heterointerface and reach the maximum value close to $2 \mathrm{GPa}$ in the dislocation core. Practically (see insertion in Fig. 3) the strain penetration depth into the substrate can be estimated approximately as $15 \ldots 20 \mathrm{~nm}$ and into the epitaxial layer - as $20 \ldots 25 \mathrm{~nm}$, although the weak residual $\sigma_{y y}$ component of the strain tensor remains at the depths of $50 \ldots 60 \mathrm{~nm}$ (Fig. 4c).

The radius of the dislocation core can be estimated from Figs 3, 4 and equals $5 \ldots 7 \mathrm{~nm}$. For values of deformation potential 12 and $11.5 \mathrm{eV}$ for $\mathrm{GaSb}$ and InAs, respectively [5], change of the substrate and epitaxial layer bandgaps at the heterointerface of dislocation core equals 3.6 and $7 \%$, respectively, and disappears at the distance close to $20 \ldots 25 \mathrm{~nm}$ from the heterointerface.

\section{Conclusions}

As a result of simulation of mechanical strains taking place in the $\mathrm{GaSb} / \mathrm{InAs}$ heterosystem in the presence of misfit dislocation network, it was found that the depth of strain penetration into the substrate reaches $15 \ldots 20 \mathrm{~nm}$ and in the epitaxial layer - up to $20 \ldots .25 \mathrm{~nm}$. The radius estimated from strain dislocation distribution is about $5 \ldots 7 \mathrm{~nm}$. It was shown that the change of the bandgap of substrate and epitaxial layer materials does not exceed 3.6 and $7 \%$, respectively.

\section{Acknowledgements}

The authors thank DSc, Professor I.V. Kurylo for his useful discussions.

\section{References}

1. M.J. Yang, C.H. Yang, B.R. Bennett, and B.V. Shanabrook, Evidence of a hybridization gap in "semimetallic" InAs/GaSb systems // Phys. Rev. Lett. 78, p. 4613-4616 (1997).

2. G.J. Conibeer, C.W. Bumby, R.J. Nicholas, and N.J. Mason, Towards a GaSb-InAs tandem junction TPV cell // Proc. of $17^{\text {th }}$ European PV Solar Energy Conf. and Exhibition, Munich, Germany, p. 175-179 (2001).

3. M.G. Mil'vidskij, V.B. Osvenskij, Structural defects in semiconductor epitaxial layers. Metallurgija, Moscow, 1985 (in Russian).

4. J.H. Van-der-Merwe, Crystal interfaces // J. Appl. Phys. 34, p. 117-127 (1963).

5. P.I. Baranskij, V.P. Klochkov, I.V. Potykevich, Semiconductor electronics. Reference book. Naukova Dumka, Kiev, 1975 (in Russian).

6. R.H. Gallagher, Finite element analysis. PrenticeHall, Inc. Englewood Cliffs, New Jersey, 1975.

7. F.R.N. Nabarro // Proc. Phys. Soc. 59, p. 256-272 (1947). 